\title{
Cathelicidin-related antimicrobial peptide modulates the severity of acute pancreatitis in mice
}

\author{
YUAN-YUAN DENG ${ }^{1}$, MUHAMMAD SHAMOON ${ }^{1}$, YUE HE $^{1}$, MADHAV BHATIA $^{2}$ and JIA SUN ${ }^{1}$ \\ ${ }^{1}$ State Key Laboratory of Food Science and Technology, School of Food Science and Technology, \\ Synergetic Innovation Center of Food Safety and Nutrition, Jiangnan University, Wuxi, Jiangsu 214122, P.R. China; \\ ${ }^{2}$ Department of Pathology, University of Otago, Christchurch 8140, New Zealand
}

Received November 2, 2015; Accepted February 4, 2016

DOI: $10.3892 / \mathrm{mmr} .2016 .5008$

\begin{abstract}
The present study aimed to investigate the immunomodulatory effects of mouse cathelicidin-related antimicrobial peptide (CRAMP) on experimental acute pancreatitis (AP). AP is a common clinical condition characterized by acute abdominal inflammation. Innate immune cells and mediators are intrinsically linked to the pathogenesis of AP. Cathelicidins are innate immunity-derived antimicrobial peptides that exert immunomodulatory effects on various host cells. However, how cathelicidins are involved and modulate the severity and inflammatory responses of AP remains unclear. In the present study, the mouse CRAMP gene-deficient $\mathrm{cnlp}^{-/-}$mice and their wild-type C57BL/6J littermates were induced with AP by multiple hourly injections of supramaximal doses of caerulein. Serum amylase levels, pancreatic myeloperoxidase activity and histological examination were performed in order to determine the disease severity and the levels of inflammatory cytokines. Disease severity and inflammatory markers were subsequently evaluated in the control mice, $c^{-1 /} p^{-C} 57 \mathrm{BL} / 6 \mathrm{~J}$ mice with AP, and wild-type C57BL/6J mice with AP. The results demonstrated that $c n l p^{-/-}$mice exhibited a more severe phenotype and inflammatory response following AP induction compared with the wild-type mice, as evidenced by increased serum amylase levels, pancreatic myeloperoxidase release, and early inflammatory mediator tumor necrosis factor- $\alpha$ production.
\end{abstract}

Correspondence to: Dr Jia Sun, State Key Laboratory of Food Science and Technology, School of Food Science and Technology, Synergetic Innovation Center of Food Safety and Nutrition, Jiangnan University, 1800 Lihu Avenue, Wuxi, Jiangsu 214122, P.R. China E-mail: jiasun@jiangnan.edu.cn

Abbreviations: AP, acute pancreatitis; AMP, antimicrobial peptide; CRAMP, cathelicidin-related antimicrobial peptide; $\mathrm{MCP}$, monocyte chemotactic protein; MPO, myeloperoxidase; IL, interleukin; TNF, tumor necrosis factor

Key words: immunomodulation, CRAMP, pancreatic inflammation, inflammatory mediators
Histological examination confirmed that CRAMP deficiency worsened the pancreatic inflammatory condition. These results indicate that CRAMP may be considered a novel modulatory mediator in mouse experimental AP.

\section{Introduction}

Acute pancreatitis (AP) is a common abdominal inflammatory disease for which a specific clinical treatment remains elusive $(1,2)$. The majority of patients with AP exhibit a mild form of the disease; however, 20-25\% of patients suffer a severe episode and consequently may develop multiple organ dysfunction syndrome, a main cause of AP-associated mortality $(3,4)$. Alcoholism and gallstones are the most common etiological factors, which lead to intrapancreatic trypsinogen activation and cellular injury of the pancreas $(2,5)$. Innate immune cells and mediators have important roles in the pathogenesis of AP $(6,7)$, and prognosis of the disease is directly associated with the intensity of inflammation. Immune cell infiltration and elevated serum levels of mediators, including tumor necrosis factor (TNF)- $\alpha$ and interleukin (IL)-1 $\beta$, are used as markers of inflammatory responses (8). Recently, antimicrobial peptides (AMPs), particularly $\alpha$-defensins, have been implicated in AP (9). AMPs are innate immunity-derived peptides, which are primarily expressed by epithelial cells and infiltrating immune cells in mammals under steady state or during inflammation (10). AP is an inflammatory disorder, and is therefore associated with altered permeability of the AMP-producing cells, thus suggesting a potential role for AMPs in this condition.

Among AMPs, cathelicidins are pleiotropic AMPs that possess broad-spectrum antimicrobial activities and have a major role in regulating local inflammation and immunity $(11,12)$. Cathelicidins are characteristically cationic and share a conserved $\mathrm{N}$-terminal pro-region, which is termed the cathelin domain, and a variable $\mathrm{C}$-terminal antimicrobial domain. A single cathelicidin is found in humans (hCAP18/LL-37) and its orthologs in the rat and mouse are rat cathelicidin-related antimicrobial peptide (CRAMP) and mouse CRAMP, respectively $(13,14)$. In addition to their antimicrobial activities, cathelicidins have been reported to exert modulatory effects on various host cells, notably epithelial 
and immune cells (15-18). Cathelicidins contribute to immune cell recruitment and activation, cytokine production, modulation of inflammatory responses during inflammatory bowel diseases and gastrointestinal inflammation $(12,18)$. However, the role of CRAMP in AP remains clear.

AP is associated with complex episodes of inflammation of the pancreatic acinar cells and distant organs. While the cellular and molecular regulatory mechanisms underlying AP pathogenesis remain to be fully elucidated for the identification of a curative treatment, exploration of novel innate immunomodulatory mediators may yield a promising outcome (19). Therefore, the present study investigated the potential effects of CRAMP on caerulein-induced experimental AP in mice. The results support a modulatory role of CRAMP in AP, and suggest CRAMP may be a potential therapeutic target for future investigation.

\section{Materials and methods}

Animals. Male C57BL/6J (Su Pu Si Biotechnology Co., Ltd., Suzhou, China) and CRAMP-deficient $c n l p^{-/}$mice (C57BL/6J background; age, 8 weeks; The Jackson Laboratory, Sacramento, CA, USA) were maintained at the Animal Housing Unit of Jiangnan University (Wuxi, China) under a controlled temperature $\left(23-25^{\circ} \mathrm{C}\right)$ and a $12 \mathrm{~h}$ light/12 h dark cycle. All of the mice were provided with standard laboratory chow and water ad libitum. All experimental protocols were approved by the Animal Ethics Committee of Jiangnan University, and were performed in accordance with the guidelines therein.

Reagents. Caerulein and tetramethylbenzidine substrate were used for enzyme-linked immunosorbent assay (ELISA) assays and were purchased from Sigma-Aldrich (St. Louis, MO, USA). Amylase and myeloperoxidase (MPO) activity measurement kits were purchased from the Jiancheng Bioengineering Institute (Nanjing, China). Mice TNF- $\alpha$ and mice monocyte chemotactic protein (MCP)-1 ELISA kits were obtained from Biolegend, Inc. (San Diego, CA, USA). All other reagents were supplied locally by the material library of Jiangnan University and were purchased from National Medicine Group Chemical Reagent Co., Ltd. (Shanghai, China).

Induction of AP. Mice were randomly assigned into the control and experimental groups $(\mathrm{n}=8)$. The groups were as follows: CRAMP gene knockout $\left(\mathrm{cnlp}^{-/}\right)$mice and wild-type C57BL/6J mice with the same genetic background were randomly assigned into the $\mathrm{cnlp}^{-/-}$control, $\mathrm{cnlp}^{-/-}$mice with AP group [AP $\left.\left(\mathrm{cnlp}^{-/}\right)\right]$and C57BL/6J mice with AP group [AP (C57)]. The mice received hourly intraperitoneal injections with normal saline or saline containing caerulein $(50 \mu \mathrm{g} / \mathrm{kg})$ for $10 \mathrm{~h}$ to induce AP. A total of $1 \mathrm{~h}$ after the final injection, the mice were sacrificed with a lethal dose of pentobarbitone sodium $(100 \mathrm{mg} / \mathrm{kg})$. The experiments were repeated three times. Blood samples were collected in sterilized centrifuge tubes, were centrifuged to separate the serum, and were stored at $-80^{\circ} \mathrm{C}$. The harvested pancreatic tissue samples were stored at $-80^{\circ} \mathrm{C}$ for subsequent measurements of MPO activity, and cytokine and chemokine levels.
Serum amylase measurements. Serum was collected by allowing the blood to coagulate at ambient temperate for $25 \mathrm{~min}$, and subsequently centrifuging the samples at $3,000 \mathrm{x}$ g for $10 \mathrm{~min}$ at $4^{\circ} \mathrm{C}$. The supernatant was then collected for analysis. An iodine-starch colorimetric method was used to measure serum amylase levels. Briefly, serum samples were incubated with $0.5 \mathrm{ml}$ pre-warmed substrate buffer for $7.5 \mathrm{~min}$ at $37^{\circ} \mathrm{C}$. Absorbance was measured,

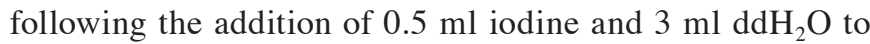
the mixture, at $660 \mathrm{~nm}$ using a UV-2450 UV-VIS spectrophotometer (Shimadzu Corporation, Kyoto, Japan).

MPO activity. MPO activity was evaluated using an MPO assay kit according to the manufacturer's protocol. Pancreatic tissues $(5 \%)$ were homogenized in $0.9 \%$ saline using an IKA homogenizer (Straufen, Germany). Absorbance was analyzed using a UV-2450 UV-VIS spectrophotometer (Shimadzu Corporation) at $460 \mathrm{~nm}$ within $10 \mathrm{~min}$ and MPO activities are expressed as units/g tissue.

ELISA assays of inflammatory mediators. Pancreatic homogenates were assayed for MCP-1 and TNF- $\alpha$ levels using sandwich ELISA kits. Previous procedures validated by our group were adopted, according to the manufacturer's protocols. Absorbance was measured at $450 \mathrm{~nm}$ within 30 min, using an automated microplate reader $\left(\right.$ Multiskan ${ }^{\mathrm{TM}}$ GO; Thermo Fisher Scientific Oy, Vantaa, Finland). TNF- $\alpha$ and MCP-1 concentrations were calculated based on the absorbance measurements, and are expressed as pg/ml.

Histological examination. Freshly harvested pancreatic samples were fixed with $4 \%$ paraformaldehyde overnight. The tissues were then washed with $\mathrm{ddH}_{2} \mathrm{O}$, dehydrated with gradient ethanol solutions and embedded in paraffin and cut into $5 \mu \mathrm{m}$ sections. The sections were subsequently stained with hematoxylin/eosin (H\&E). Pancreatic injury was examined under a DM2000 light microscope (Leica Microsystems $\mathrm{GmbH}$, Wetzlar, Germany) at x200 magnification, and was evaluated based on acinar cell injury, inflammatory cell infiltration and structural changes, which are markers of tissue damage and inflammation.

Statistical analysis. Statistical analysis was performed by independent t-test to determine if there was a difference between MPO activity levels in the control and AP (C57) group, AMY levels between AP ( $\left.\mathrm{cnlp}^{-/}\right)$and AP (C57) groups. When multiple comparisons were made, by one-way analysis of variance using GraphPad Prism (version 5; GraphPad Software Inc., San Diego, CA, USA). Tukey's honest significant difference test was performed as a post-hoc test. $\mathrm{P}<0.05$ were considered to indicate a statistically significant difference.

\section{Results}

Effects of CRAMP deficiency on serum amylase levels during $A P$. Serum amylase is measured as a sensitive biochemical marker of AP, which is released due to pancreatic acinar cell damage. Serum amylase levels were elevated in the wild-type $(\mathrm{P}<0.001)$ and $c n l p^{-/}$AP $(\mathrm{P}<0.001)$ mice compared with in 


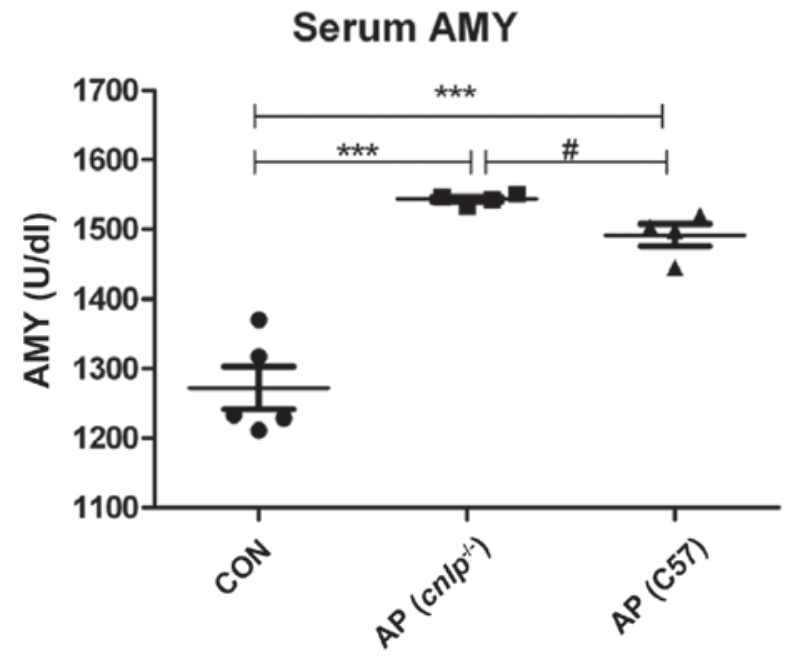

Figure 1. Effects of cathelicidin-related antimicrobial peptide deficiency $\left(\mathrm{cnlp}^{-/}\right)$on serum amylase (AMY) levels. Data are presented as the mean + standard error of the mean. ${ }^{* * * *} \mathrm{P}<0.001$ caerulein-treated mice vs. control; ${ }^{\# P}<0.05$ caerulein-treated cnlp ${ }^{-/}$mice vs. caerulein-treated C57BL/6J mice. AP, acute pancreatitis; $\mathrm{CON}$, control; $\mathrm{C} 57, \mathrm{C} 57 \mathrm{BL} / 6 \mathrm{~J}$ wild-type mice.

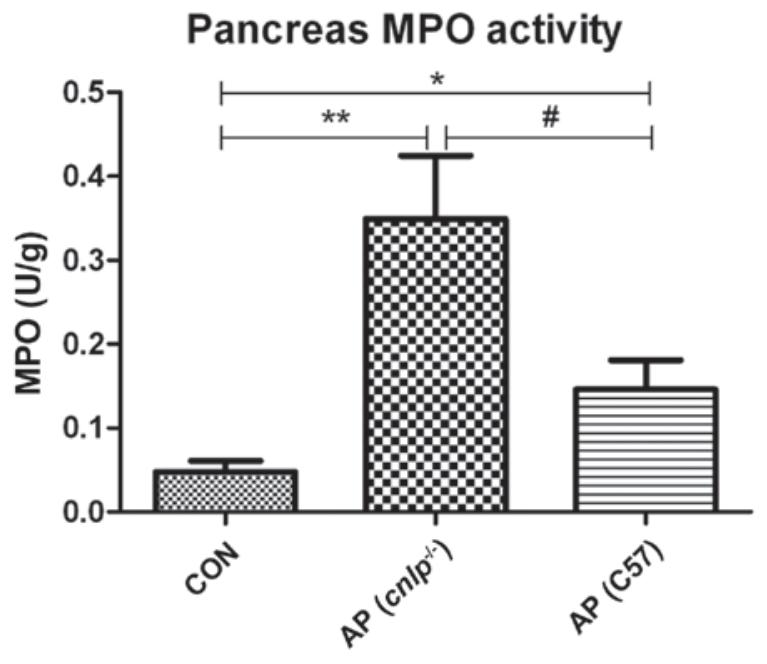

Figure 2. Effects of cathelicidin-related antimicrobial peptide deficiency $\left(\mathrm{cnlp}^{-/}\right)$on pancreatic myeloperoxidase (MPO) levels. Data are presented as the mean + standard error of the mean. ${ }^{*} \mathrm{P}<0.05$ vs. the control mice $(\mathrm{CON})$; ${ }^{* *} \mathrm{P}<0.01$ vs. CON; ${ }^{\#} \mathrm{P}<0.05$ vs. caerulein-treated $\mathrm{cnlp}^{-/-}$mice. AP, acute pancreatitis; C57, C57BL/6J wild-type mice.
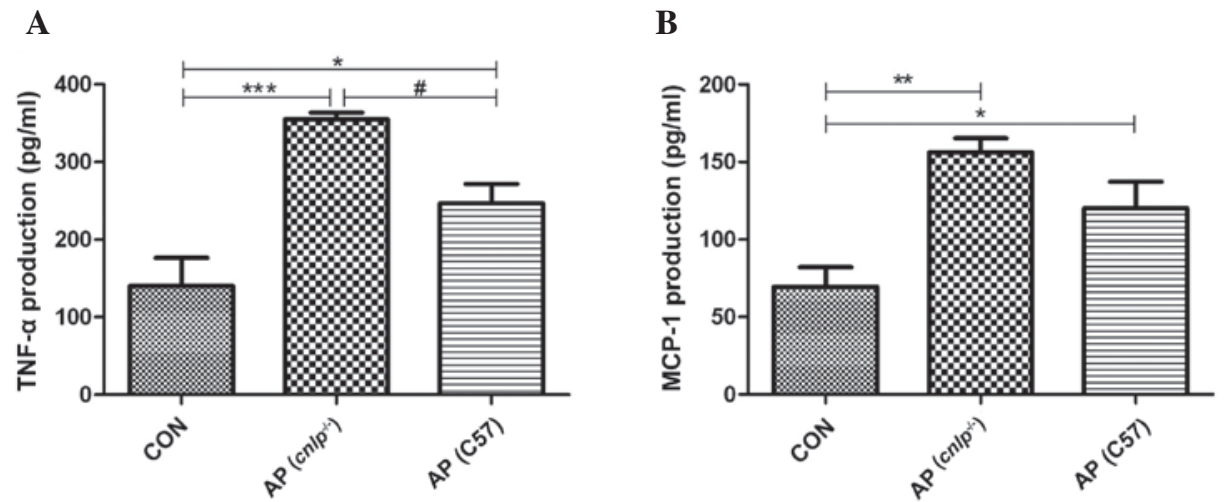

Figure 3. Effects of cathelicidin-related antimicrobial peptide deficiency $\left(\mathrm{cnlp}^{-/}\right)$on the pancreatic production of inflammatory mediators (A) tumor necrosis factor (TNF) $-\alpha$ and (B) monocyte chemotactic protein (MCP)-1. Data are presented as the mean + standard error of the mean. ${ }^{*} \mathrm{P}<0.05 \mathrm{vs}$. the control mice $(\mathrm{CON}) ;{ }^{* *} \mathrm{P}<0.01$ and ${ }^{* * * *} \mathrm{P}<0.001$ vs. CON; ${ }^{*} \mathrm{P}<0.05$ vs. caerulein-treated cnlp ${ }^{-/}$mice. AP, acute pancreatitis; C57, C57BL/6J wild-type mice.

the control mice. In addition, CRAMP-deficient mice exhibited more pronounced serum amylase levels compared with the wild-type mice $(\mathrm{P}<0.05$; Fig. 1). These findings suggest that CRAMP may have a beneficial role in protecting mice against AP.

Effects of CRAMP deficiency on pancreatic MPO release. Pancreatic MPO levels were evaluated as an indicator of neutrophil infiltration into tissues during AP. Caerulein hyperstimulation resulted in increased pancreatic MPO levels in the wild-type $(\mathrm{P}<0.05)$ and $c n l p^{-/}$mice $(\mathrm{P}<0.01$; Fig. 2$)$. This increase in MPO was more significant in the pancreatic tissues of the $\mathrm{cnlp}^{-/-}$mice compared with the wild-type mice $(\mathrm{P}<0.05$; Fig. 2). These results suggest that CRAMP may be involved in early neutrophil recruitment to the pancreas and regulating MPO activities during AP.

Effects of CRAMP deficiency on pancreatic production of inflammatory mediators. As AP propagates it is associated with enhanced production of inflammatory cytokines and chemokines at the tissue level. Therefore, the present study examined the levels of known early mediators of AP, including TNF- $\alpha$ and MCP-1. As shown in Fig. 3A and B, experimental AP is associated with increased levels of pancreatic TNF- $\alpha$ $(\mathrm{P}<0.001)$ and MCP-1 $(\mathrm{P}<0.01)$. A further increase in TNF- $\alpha$ levels (all $\mathrm{P}<0.05$ ), but not MCP-1 levels, was observed in the pancreas of $\mathrm{cnlp}^{-/-}$mice compared with in the C57BL/6J wild-type AP mice (Fig. 3A and B). These results indicate that CRAMP selectively modulates pancreatic cytokine production during AP.

Effects of CRAMP deficiency on tissue damage during AP. Pancreatic injury in mice with AP was evaluated by H\&Ehistological examination. The wild-type and CRAMP-deficient $\mathrm{cnlp}^{-/-}$mice induced with caerulein hyperstimulation exhibited pronounced pancreatic edema, enhanced neutrophil infiltration into the pancreas, and pancreatic morphological injuries, as compared with the control mice (Fig. 4A-C). 


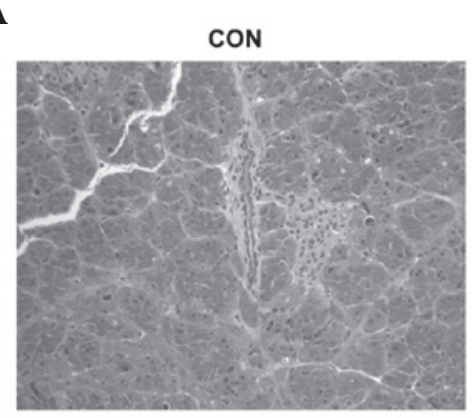

B

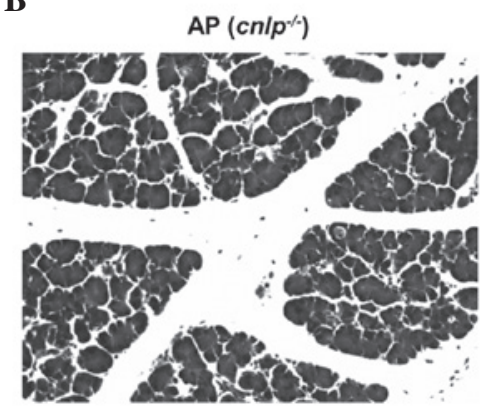

C

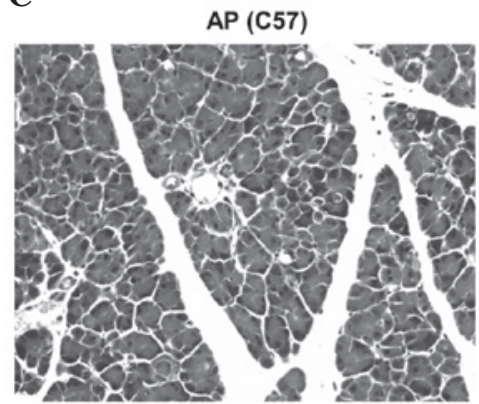

Figure 4. Histological examination of tissue damage in the cathelicidin-related antimicrobial peptide-deficient (cnlp ${ }^{-1}$ ) and wild-type C57BL/6J mice (C57) with acute pancreatitis (AP) using hematoxylin/eosin staining. Representative images (magnification, 200x) from each group from two independent experiments are presented. (A) Wild-type control mice (B) $c n l p^{-1}$ mice with AP (C) wild-type mice with AP.

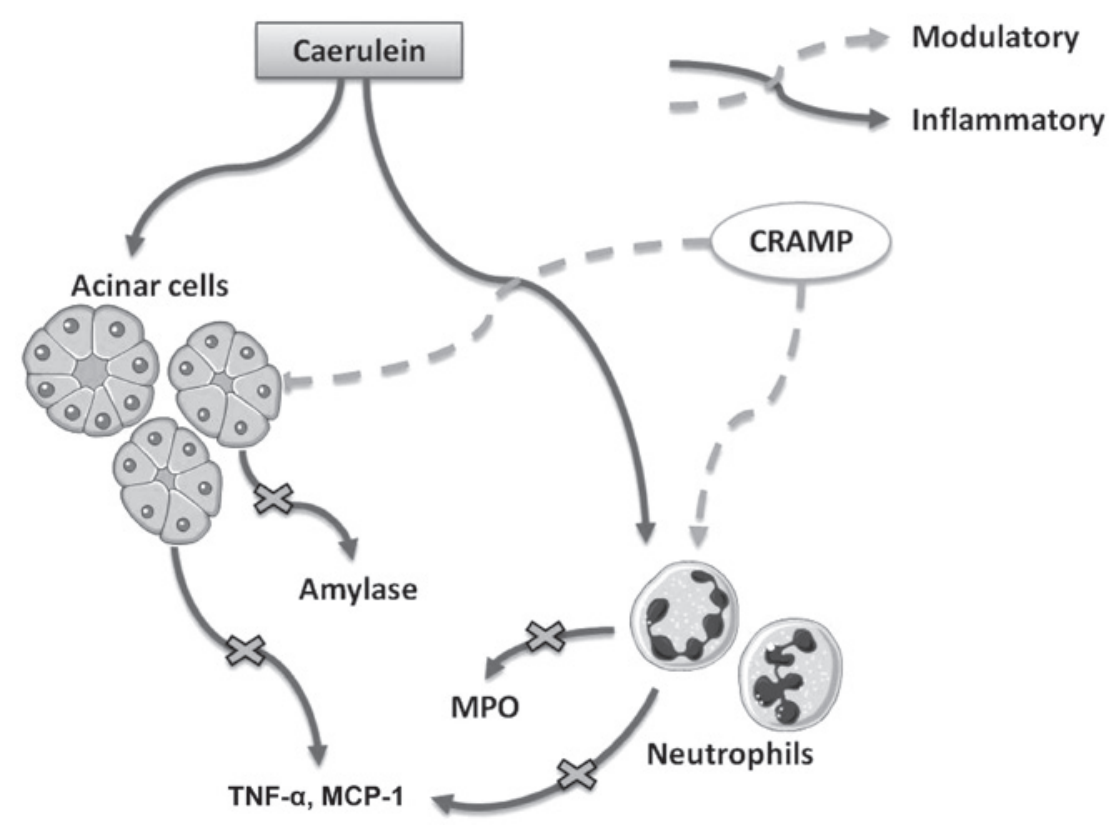

Figure 5. Graphical summary of the modulatory effects of cathelicidin-related antimicrobial peptide (CRAMP) on caerulein-induced experimental acute pancreatitis (AP). CRAMP modulates amylase release from acinar cells, myeloperoxidase (MPO) release from neutrophils, and the production of inflammatory cytokines during AP. TNF- $\alpha$, tumor necrosis factor- $\alpha$; MCP-1, monocyte chemotactic protein- 1.

Furthermore, pancreatic injury in the $c n l p^{-/}$mice (Fig. 4B) was more severe than in the wild-type mice (Fig. 4C). This histological analysis confirmed that CRAMP alleviates pancreatic injury and inflammation.

\section{Discussion}

The present study demonstrated that mouse CRAMP is a novel modulatory mediator during AP, which is particularly associated with local pancreatic inflammation. The $c n l p^{-1}$ C57BL/6J mice developed a more severe phenotype and inflammatory responses, as compared with their wild-type littermates induced with AP. In addition, the $\mathrm{cnlp}^{-1-} \mathrm{C} 57 \mathrm{BL} / 6 \mathrm{~J}$ mice exhibited more pronounced serum amylase levels, pancreatic MPO release and TNF- $\alpha$ production than the wild-type mice. These data indicated that CRAMP may have a modulatory role in experimental AP.

A cascade of cellular events, including activation of intra-acinar enzymes, release of inflammatory mediators and cytokines, acinar cell apoptosis, and pancreatic microcirculation disorder have been reported to underlie the pathogenesis of AP (20-23). However, AP pathogenesis remains to fully elucidated. AMPs represent novel mediators of the condition. Intestinal $\alpha$-defensin ( $\alpha$-defensin-5 and $\alpha$-defensin-7) levels, but not CRAMP, were shown to be elevated in aged rats with AP, and were associated with more severe local inflammation (9). The role of cathelicidins (human LL-37 and mouse CRAMP) has been particularly documented in other inflammatory processes (24); however, its role is unknown in AP. Therefore the present study investigated cathelicidins as a potential immunomodulatory mediator in a mouse model of experimental AP, using CRAMP-deficient $c n l p^{-/-}$mice.

The results of the present study demonstrated that cnlp $p^{-/}$mice with AP exhibited more pronounced acinar cell injury, as measured by serum amylase levels, MPO activity and TNF- $\alpha$ production, as compared with the wild-type AP mice. Immune cell infiltration into the tissues and 
production of inflammatory cytokines (chemokines) are key pathological events that determine, to a large extent, disease severity $(25,26)$. During AP, neutrophils are the first-line innate immune cells recruited to the pancreas. TNF- $\alpha$, which is derived predominantly from activated macrophages, acts via cell membrane-bound receptors (27) to induce proinflammatory gene expression of IL-1, IL-6, IL-8 and itself, thereby causing pancreatic tissue necrosis and further migration of leukocytes (28). The prototypic CC chemokine MCP-1 is an early mediator associated with chemo-attraction of macrophages and further propagation of local to systemic inflammation (29). Although both TNF- $\alpha$ and MCP-1 are significantly elevated in the pancreas of $\mathrm{cnlp}^{-/-}$and wild-type AP mice (Fig. 3), only TNF- $\alpha$ levels were significantly higher in the $\mathrm{cnlp}^{-/-}$mice than the wild-type mice, thus suggesting that the regulatory effects of CRAMP may be selective towards TNF- $\alpha$-producing cells in the pancreas.

The present study detected a more resistant phenotype during AP due to the C57BL/6J genetic background of $\mathrm{cnlp}^{-1-}$ and wild-type mice. Different mouse strains have exhibited varied susceptibility to caerulein-induced AP. Serum amylase and $\mathrm{TNF}-\alpha$ production in $\mathrm{BALB} / \mathrm{c}$ mice are significantly higher than in C57BL/6J mice upon AP induction (unpublished data). Despite this intrinsic variation of responses, CRAMP deficiency still worsens pancreatic inflammatory conditions, further suggesting its modulatory role in AP.

In conclusion, the present study is the first, to the best of our knowledge, to demonstrate that cathelicidins exert immune regulatory effects on AP in mice (Fig. 5). In addition, the present study provides novel evidence for the effective prevention and treatment of AP in clinical practice.

\section{Acknowledgements}

The present study was supported by funds from the National Natural Science Foundation of China (grant nos. 31400779 and 31570915; National Youth 1000 Talents Plan), the Provincial Natural Science Foundation of Jiangsu (grant no. BK20130133), and the Jiangsu Provincial Shuang Chuang Innovator Plan, Jiangsu Province Recruitment Plan for High-level, Innovative and Entrepreneurial Talents and Jiangsu Province 'Six Summit Talents' Program (grant no. 2014-SWYY-035) to J.S.

\section{References}

1. Bell D, Keane MG and Pereira SP: Acute pancreatitis. Medicine 43: 174-181, 2015.

2. Petrov M: Nutrition, inflammation, and acute pancreatitis. ISRN Inflamm 2013: 341410, 2013.

3. Kylänpää L, Rakonczay Z Jr and O'Reilly DA: The clinical course of acute pancreatitis and the inflammatory mediators that drive it. Int J Inflam 2012: 360685, 2012.

4. Johnson CD and Abu-Hilal M: Persistent organ failure during the first week as a marker of fatal outcome in acute pancreatitis. Gut 53: 1340-1344, 2004.

5. DiMagno MJ and DiMagno EP: New advances in acute pancreatitis. Curr Opin Gastroenterol 23: 494-501, 2007.

6. Abdulla A, Awla D, Thorlacius H and Regnér S: Role of neutrophils in the activation of trypsinogen in severe acute pancreatitis. J Leukoc Biol 90: 975-982, 2011.
7. Akinosoglou $\mathrm{K}$ and Gogos $\mathrm{C}$ : Immune-modulating therapy in acute pancreatitis: Fact or fiction. World J Gastroenterol 20: 15200-15215, 2014.

8. Bhatia M, Neoptolemos JP and Slavin J: Inflammatory mediators as therapeutic targets in acute pancreatitis. Curr Opin Investig Drugs 2: 496-501, 2001.

9. Cunha DM, Koike MK, Barbeiro DF, Barbeiro HV, Hamasaki MY, Coelho Neto GT, Machado MC and da Silva FP: Increased intestinal production of $\alpha$-defensins in aged rats with acute pancreatic injury. Exp Gerontol 60: 215-219, 2014.

10. Gallo RL and Hooper LV: Epithelial antimicrobial defence of the skin and intestine. Nat Rev Immunol 12: 503-516, 2012.

11. Hiemstra PS: Defensins and cathelicidins in inflammatory lung disease: Beyond antimicrobial activity. Biochem Soc Trans 34: 276-278, 2006

12. Hilchie AL, Wuerth $\mathrm{K}$ and Hancock RE: Immune modulation by multifaceted cationic host defense (antimicrobial) peptides. Nat Chem Biol 9: 761-768, 2013.

13. Sørensen OE, Follin P, Johnsen AH, Calafat J, Tjabringa GS Hiemstra PS and Borregaard N: Human cathelicidin, hCAP-18, is processed to the antimicrobial peptide LL-37 by extracellular cleavage with proteinase 3. Blood 97: 3951-3959, 2001.

14. Zanetti M, Gennaro R and Romeo D: Cathelicidins: A novel protein family with a common proregion and a variable C-terminal antimicrobial domain. FEBS Lett 374: 1-5, 1995.

15. Zanetti M: The role of cathelicidins in the innate host defenses of mammals. Curr Issues Mol Biol 7: 179-196, 2005.

16. Shaykhiev R, Beisswenger C, Kändler K, Senske J, Püchner A, Damm T, Behr J and Bals R: Human endogenous antibiotic LL-37 stimulates airway epithelial cell proliferation and wound closure. Am J Physiol Lung Cell Mol Physiol 289: L842-L848, 2005.

17. Nagaoka I, Tamura $\mathrm{H}$ and Hirata $\mathrm{M}$ : An antimicrobial cathelicidin peptide, human CAP18/LL-37, suppresses neutrophil apoptosis via the activation of formyl-peptide receptor-like 1 and P2X7. J Immunol 176: 3044-3052, 2006.

18. Wehkamp J, Harder J, Weichenthal M, Mueller O, Herrlinger KR, Fellermann K, Schroeder JM and Stange EF: Inducible and constitutive beta-defensins are differentially expressed in Crohn's disease and ulcerative colitis. Inflamm Bowel Dis 9: 215-223, 2003.

19. Dawra R, Sah RP, Dudeja V, Rishi L, Talukdar R, Garg P and Saluja AK: Intra-acinar trypsinogen activation mediates early stages of pancreatic injury but not inflammation in mice with acute pancreatitis. Gastroenterology 141: 2210-2217, 2011.

20. Bhatia M: Apoptosis versus necrosis in acute pancreatitis. Am J Physiol Gastrointest Liver Physiol 286: G189-G196, 2004.

21. Sah RP and Saluja A: Molecular mechanisms of pancreatic injury. Curr Opin Gastroenterol 27: 444-451, 2011.

22. Sah RP, Dawra RK and Saluja AK: New insights into the pathogenesis of pancreatitis. Curr Opin Gastroenterol 29: 523-530, 2013.

23. Criddle DN, McLaughlin E, Murphy JA, Petersen OH and Sutton R: The pancreas misled: Signals to pancreatitis. Pancreatology 7: 436-446, 2007.

24. Meguro S, Tomita M, Katsuki T, Kato K, Oh H, Ainai A, Ito R, Kawai T, Itoh $\mathrm{H}$ and Hasegawa $\mathrm{H}$ : Plasma antimicrobial peptide LL-37 level is inversely associated with HDL cholesterol level in patients with type 2 diabetes mellitus. Int J Endocrinol 2014: 703696, 2014.

25. Xue J, Sharma V and Habtezion A: Immune cells and immune-based therapy in pancreatitis. Immunol Res 58: 378-386, 2014.

26. Mayerle J, Dummer A, Sendler M, Malla SR, van den Brandt C, Teller S, Aghdassi A, Nitsche C and Lerch MM: Differential roles of inflammatory cells in pancreatitis. J Gastroenterol Hepatol 27 (Suppl 2): 47-51, 2012.

27. Bhatia M, Brady M, Shokuhi S, Christmas S, Neoptolemos JP and Slavin J: Inflammatory mediators in acute pancreatitis. JPathol 190: 117-125, 2000.

28. Norman JG, Fink GW and Franz MG: Acute pancreatitis induces intrapancreatic tumor necrosis factor gene expression. Arch Surg 130: 966-970, 1995.

29. Sun J and Bhatia M: Blockade of neurokinin-1 receptor attenuates $\mathrm{CC}$ and $\mathrm{CXC}$ chemokine production in experimental acute pancreatitis and associated lung injury. Am J Physiol Gastrointest Liver Physiol 292: G143-G153, 2007. 